Published in final edited form as:

Br J Dermatol. 2013 March ; 168(3): 660-661. doi:10.1111/j.1365-2133.2012.11211.x.

\title{
The effect of weight loss surgery on the severity of psoriasis
}

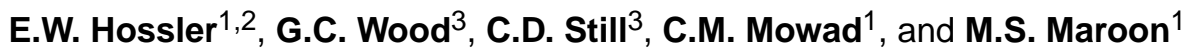 \\ E.W. Hossler: ewhossler@geisinger.edu \\ ${ }^{1}$ Departments of Dermatology, Geisinger Obesity Institute and Center for Nutrition and Weight \\ Management, Geisinger Medical Center, Danville, PA 17821, U.S.A \\ ${ }^{2}$ Departments of Pathology, Geisinger Obesity Institute and Center for Nutrition and Weight \\ Management, Geisinger Medical Center, Danville, PA 17821, U.S.A \\ ${ }^{3}$ Departments of Geisinger Center for Health Research and Geisinger Obesity Institute and \\ Center for Nutrition and Weight Management, Geisinger Medical Center, Danville, PA 17821, \\ U.S.A
}

Madam, Obesity is a growing problem; in 2007-2008, 68\% of U.S. adults were obese or overweight [body mass index (BMI) $\geq 25.0 \mathrm{~kg} \mathrm{~m}^{-2}$ ], ${ }^{1}$ and the prevalence of childhood obesity has more than tripled since the 1960 s. $^{2}$ Psoriasis is more common in overweight individuals, and increasing BMI is associated not only with greater extent of psoriasis but also with refractory disease, ${ }^{3}$ including lack of response to biological agents. ${ }^{4}$ Recent publications reporting complete remission of severe psoriasis following bariatric surgery ${ }^{5}{ }^{7}$ prompted us to perform a retrospective review of our bariatric surgery population to investigate the effects of such surgery on patients with psoriasis.

An electronic search of Geisinger Health System's electronic medical record (EMR), serving over 2.6 million patients, was used to identify adults aged 18 years or older with a diagnosis of psoriasis or psoriatic arthritis and a procedure code documenting weight loss surgery between January 2004 and July 2009. For those meeting the inclusion criteria, contact information and demographic data were extracted and a telephone survey was conducted, after institutional approval.

An opt-out letter was sent to eligible patients. We then called the remaining patients a maximum of three times during the day and evening. The interviewer received permission to conduct the interview and obtained information regarding duration of skin disease, treatment, family history and change in skin disease following surgery.

The primary outcome measure was the percentage of patients who reported improvement in psoriasis after surgery. In secondary analyses, changes in psoriasis since surgery and a change in psoriasis treatment class [categorized as none, only topical, or systemic (including ultraviolet radiation)] were correlated with patient demographics.

We identified 104 patients, none of whom opted out of the study. Fifty-four patients (52\%) were contacted by telephone and 34 (63\%) of these completed the interview. Twenty patients

Conflicts of interest: none declared. 
were excluded because they denied having psoriasis $(n=8)$, they refused to participate $(n=$ $3)$, or their psoriasis began after $(n=2)$ or cleared prior to surgery $(n=1)$. Six additional patients were excluded because we were unable to confirm bariatric surgery in the EMR.

Baseline demographic data are given in Table 1. Most patients (88\%) were female, with a mean age of 50 years and a mean duration of psoriasis of 20 years. All patients were obese (mean presurgical weight, $132 \mathrm{~kg}$; mean BMI, $48.5 \mathrm{~kg} \mathrm{~m}^{-2}$ ). Thirty patients (88\%) underwent Roux-en-Y gastric bypass surgery.

Twenty-one patients (62\%) reported improvement in psoriasis after surgery, nine (26\%) reported no change and four (12\%) reported worsening. Of those reporting improvement, most noted a decrease in redness, scaling, lesion thickness and itch. Three patients who experienced initial improvement subsequently worsened.

A significant downgrade in psoriasis treatment was noted after surgery $(P=0.046)$. Four patients went from systemic to topical therapy, and seven went from topical therapy to none. Only two patients (6\%) reported an escalation of therapy.

Age at the time of surgery was significantly associated with a change in psoriasis after surgery ( $P=0.039$, Table 2 ). Those who worsened tended to be younger (mean age 38.5 years), whereas those who improved were older (mean age 52.7 years). All of the men ( $n=$ $4,100 \%)$ reported improvement but this was not statistically significant $(P=0.416)$. No other characteristic was statistically associated with self-reported improvement in psoriasis $(P>0.05)$. A similar change in BMI was noted in the three patients who initially improved and then worsened (data not shown). Post hoc analysis demonstrated that subjects aged $<45$ years at surgery and with a family history of psoriasis were less likely to report improvement after surgery compared with all others $(P=0.007)$.

Almost two-thirds of patients with psoriasis who underwent weight-loss surgery reported a postoperative improvement in psoriasis. Previous to this report, only case reports of psoriasis improving after bariatric surgery had been published. ${ }^{5}{ }^{7}$ Our study provides further data that psoriasis may improve after weight-loss surgery, and also provides some preliminary evidence that weight-loss surgery may reduce the need for medical therapy for psoriasis.

The reasons why psoriasis improves with weight loss are unknown, but reduction of the systemic inflammation associated with obesity is likely pivotal. Obesity is associated with higher levels of circulating tumour necrosis factor a (TNF- $a),{ }_{9}^{8}$ and bariatric surgery has been shown to lower TNF-a expression within adipose tissue.

Psoriasis is, to some extent, a genetically inherited, familial disease. A trend was noted towards positive family history and lack of improvement in psoriasis after bariatric surgery, suggesting that genetically inherited psoriasis may not be as responsive to weight loss.

Our study has a number of limitations including reliance on patient recall, lack of dermatologist confirmation of psoriasis, and lack of quantitative assessment of disease burden. Our study also does not prove causality. An improvement in psoriasis may be due to a number of different factors such as discontinuation of antihypertensive medications or 
increased ambient sunlight. Prospective trials are needed to further clarify our observations and to clarify the role of surgical weight loss in the management of psoriasis.

\section{Acknowledgments}

Funding sources: none.

\section{References}

1. Flegal KM, Carroll MD, Ogden CL, Curtin LR. Prevalence and trends in obesity among US adults, 1999-2008. JAMA. 2010; 303:235-41. [PubMed: 20071471]

2. Ogden CL, Carroll MD, Curtin LR, et al. Prevalence of high body mass index in US children and adolescents, 2007-2008. JAMA. 2010; 303:242-9. [PubMed: 20071470]

3. Sakai R, Matsui S, Fukushima M, et al. Prognostic factor analysis for plaque psoriasis. Dermatology. 2005; 211:103-6. [PubMed: 16088154]

4. Bremmer S, Van Voorhees AS, Hsu S, et al. Obesity and psoriasis: from the Medical Board of the National Psoriasis Foundation. J Am Acad Dermatol. 2010; 63:1058-69. [PubMed: 20692722]

5. Higa-Sansone G, Szomstein S, Soto F, et al. Psoriasis remission after laparoscopic Roux-en-Y gastric bypass for morbid obesity. Obes Surg. 2004; 14:1132-4. [PubMed: 15479606]

6. de Menezes Ettinger JE, Azaro E, de Souza CA, et al. Remission of psoriasis after open gastric bypass. Obes Surg. 2006; 16:94-7. [PubMed: 16417765]

7. Hossler EW, Maroon MS, Mowad CM. Gastric bypass surgery improves psoriasis. J Am Acad Dermatol. 2011; 65:198-200. [PubMed: 20655127]

8. Eckel RH, Grundy SM, Zimmet PZ. The metabolic syndrome. Lancet. 2005; 365:1415-28. [PubMed: 15836891]

9. Hotamisligil GS, Arner P, Caro JF, et al. Increased adipose tissue expression of tumor necrosis factor-alpha in human obesity and insulin resistance. J Clin Invest. 1995; 95:2409-15. [PubMed: $7738205]$ 
Table 1

Characteristics of study population who completed the telephone survey at the time of surgery

\begin{tabular}{llr}
\hline Subjects with psoriasis at the time of surgery & & $\mathbf{n}=\mathbf{3 4}$ \\
\hline Sex & Male, $\mathrm{n}(\%)$ & $4(12 \%)$ \\
& Female, $\mathrm{n}(\%)$ & $30(88 \%)$ \\
Age (years) & Mean (SD) & $49.8(10.7)$ \\
& Median (range) & $48(27,71)$ \\
Family history of psoriasis & Yes, $\mathrm{n}(\%)$ & $17(52 \%)$ \\
& No, $\mathrm{n}(\%)$ & $16(48 \%)$ \\
& Unknown & $\mathrm{n}=1$ \\
Time with psoriasis (years) & Mean (SD) & $20.0(16.8)$ \\
Psoriasis treatment prior to surgery & Median (range) & $17(1,55)$ \\
& Middleical, $\mathrm{n}(\%)$ & $22(65 \%)$ \\
Type of bariatric surgery & Systemic, $\mathrm{n}(\%)$ & $10(29 \%)$ \\
& None, $\mathrm{n}(\%)$ & $2(6 \%)$ \\
& Roux-en-Y, $\mathrm{n}(\%)$ & $30(88 \%)$ \\
Body mass index at surgery $\left(\mathrm{kg} \mathrm{m}^{-2}\right)$ & Gastric band, $\mathrm{n}(\%)$ & $3(12 \%)$ \\
& Unknown, $\mathrm{n}(\%)$ & $\mathrm{n}=1$ \\
Weight at surgery $(\mathrm{kg})$ & Mean (SD) & $132(27)$ \\
& Median (range) & $124(87,205)$ \\
& Mean (SD) & $165(8.4)$ \\
& Median (range) & $165(147,190.5)$ \\
& Mean (SD) & $48.5(8.5)$ \\
& & $48.4(35.3,70.5)$ \\
\hline
\end{tabular}

Br J Dermatol. Author manuscript; available in PMC 2016 June 07. 


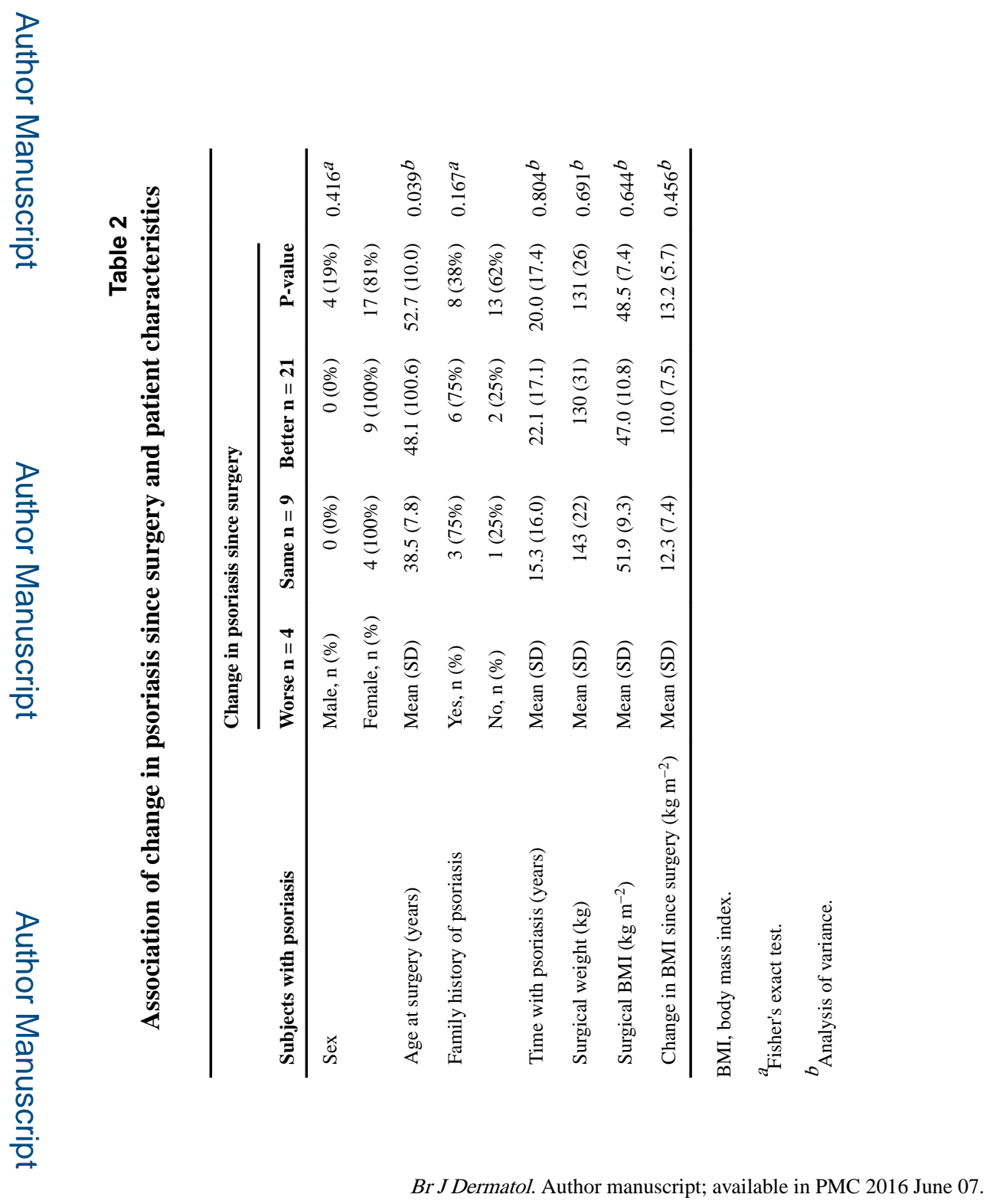

